\title{
Synthesis and antibacterial evaluation of some teicoplanin pseudoaglycon derivatives containing alkyl- and arylthiosubstituted maleimides
}

\author{
Magdolna Csávás ${ }^{1}$, Adrienn Miskovics ${ }^{1}$, Zsolt Szúcs ${ }^{1}$, Erzsébet Rőth ${ }^{1}$, Zsolt L Nagy ${ }^{1}$, Ilona Bereczki ${ }^{1}$, \\ Mihály Herczeg ${ }^{1}$, Gyula Batta ${ }^{2}$, Éva Nemes-Nikodém ${ }^{3}$, Eszter Ostorházi ${ }^{3}$, Ferenc Rozgonyi ${ }^{3}$, Anikó Borbás ${ }^{1}$ and \\ Pál Herczegh ${ }^{1}$ Dedicated to the memory of Professor Maria N Preobrazhenskaya
}

Bis-alkylthio maleimido derivatives have been prepared from teicoplanin pseudoaglycon by reaction of its primary amino group with Nethoxycarbonyl bis-alkylthiomaleimides. Some of the new derivatives displayed excellent antibacterial activity against resistant bacteria.

The Journal of Antibiotics (2015) 68, 579-585; doi:10.1038/ja.2015.33; published online 1 April 2015

\section{INTRODUCTION}

Glycopeptide antibiotics exert their antibacterial activity by inhibiting two sequential enzymatic reactions-transglycosylation and transpeptidation-in the bacterial cell-wall biosynthesis. The antibiotics recognize and tightly bind to the L-Lys-D-Ala-D-Ala termini of peptidoglycan precursors at the external side of the developing bacterial membrane. In this way transglycosylation and transpeptidation are physically prevented, arresting cell-wall elongation and crosslinking and leading to cell lysis. ${ }^{1}$ Due to the lack of cross-resistance to other antibacterial drugs, the glycopeptide antibiotics have become first-line drugs for the treatment of life-threatening multi-drug resistant infections by Gram-positive bacteria. ${ }^{2}$

The emergence and spread of glycopeptide-resistant enterococci and glycopeptide intermediate-resistant Staphylococcus aureus, as well as teicoplanin-resistant Staphylococcus haemolyticus ${ }^{3}$ present a serious global challenge and have led to renewed interest in the development of novel, effective and safe antibacterials including new derivatives of glycopeptide antibiotics. ${ }^{4-6}$

Inspired by the high activity of the semisynthetic lipoglycopeptide antibiotics telavancin, ${ }^{7}$ dalbavancin ${ }^{8}$ and oritavancin ${ }^{9}$ against vancomycin-resistant bacteria, we have started a program to produce new antibiotics by introducing lipophilic subtituents to the primary amino function of ristocetin aglycon and of teicoplanin pseudoaglycon. Applying various approaches including squaric acid conjugation method, azide-alkyne cycloaddition reaction or three-component isoindole formation, we have prepared a large set of new derivatives exhibiting high antibacterial ${ }^{10-13}$ and, in some cases, robust antiinfluenza virus activity. ${ }^{14-17}$
Recently, Caddick, Baker and coworkers ${ }^{18-21}$ reported on applications of 3,4-dibromomaleimides for site-specific protein modification and bioconjugation. The method is based on addition-elimination reaction of thiols to the bromomaleimides leading to regeneration of the double bond resulting in thiomaleimide products (Scheme 1). Last year the group of Caddick and Baker published a simple method for the synthesis of $\mathrm{N}$-functionalised bromo- and thiomaleimides through the corresponding $N$-ethoxycarbonyl maleimide derivatives. ${ }^{22}$ Applying these recent results of maleimide chemistry we describe here derivatisation of teicoplanin pseudoaglycon with thiomaleimide substituents carrying two lipophilic alkyl or aryl sulfide side chains.

\section{RESULTS AND DISCUSSION}

Dibromomaleimide (1) that can be obtained by simple bromination of maleimide ${ }^{23}$ has been allowed to react with a range of thiols including the 6-thio-D-galactose derivative $\mathbf{2 a}$, thiophenol $\mathbf{2} \mathbf{b}$, phenylmethanethiol 2c, dodecanethiol 2d, octanethiol 2e, propanethiol $2 \mathrm{f}$ and $t$-butyl mercaptane $\mathbf{2 h}$, representing a series of substituents of different lipophilicity.

The obtained sulfides $\mathbf{3 a - g}$ have been then ethoxycarbonylated with ethyl chloroformate in the presence of potassium carbonate to provide $\mathbf{5 a} \mathbf{- g}$, ready for a reaction with a primary amino group (Scheme 2). Direct methoxycarbonylation ${ }^{22}$ of dibromomaleimide offers an alternative route for the synthesis of the targeted $N$-functionalized dithiomaleimide as it is illustrated by the synthesis of $\mathbf{6 g}$. We tested this route with several thiols such as $\mathbf{2} \mathbf{d}-\mathbf{2} \mathbf{g}$, however, the sulfide formation showed low efficacy in all cases.

\footnotetext{
${ }^{1}$ Department of Pharmaceutical Chemistry, University of Debrecen, Debrecen, Hungary; ${ }^{2}$ Department of Organic Chemistry, University of Debrecen, Debrecen, Hungary and ${ }^{3}$ Department of Dermatology, Venerology and Dermatooncology, Microbiology Laboratory, Semmelweis University, Budapest, Hungary Feceased on 25 December 2014

Correspondence: Professor A Borbás or Professor P Herczegh, Department of Pharmaceutical Chemistry, University of Debrecen, Egyetem tér 1, P.O.Box 70, Debrecen H-4010, Hungary.

E-mail: borbas.aniko@pharm.unideb.hu or herczeghp@gmail.com

Received 19 December 2014; revised 18 February 2015; accepted 27 February 2015; published online 1 April 2015
} 
Next, teicoplanin pseudoaglycon $7^{16}$ has been reacted with $\mathrm{N}$-ethoxycarbonyl maleimides $\mathbf{5 a}-\mathbf{g}$ and $\mathbf{6 g}$ in the presence of triethylamine (Table 1). In these reactions bis-alkyl- or arylthiomaleimide $\mathbf{8} \mathbf{a}-\mathbf{f}$ were formed in moderate yields, together with the $\mathrm{N}$ alkoxycarbonyl derivatives of the teicoplanin pseudoaglycon (9 and 10). The formation of $\mathbf{9}$ and $\mathbf{1 0}$ can be explained by the steric hindrance of the amino function of 7 . In the case of $5 \mathrm{~g}$ and $\mathbf{6 g}$, the undesired carbamate derivatives $\mathbf{9}$ and $\mathbf{1 0}$ were dominantly formed, probably due to the presence of bulky $t$-butyl substituents of the reagents.

Antibacterial activity of maleimido-teicoplanin-pseudoaglycons was evaluated on a panel of Gram-positive bacteria (Table 2). The Dgalactose-containing $\mathbf{8 a}$, the bis-phenylthio derivative $\mathbf{8 b}$ and the bisbenzylthio derivative $\mathbf{8 c}$ displayed similar activities than teicoplanin pseudoaglycon 7 with one exception: the maleimido compounds $8 \mathbf{8}-\mathbf{c}$ were active against Enterococcus faecalis 15376 having vanA resistance gene while teicoplanin and 7 were completely inactive against this bacterium strain.

The detected antibacterial activities of $\mathbf{8 d}, \mathbf{8 e}$ and $\mathbf{8} \mathbf{f}$ were related to the length of the alkyl chain substituents of their maleimide residues. The bis-dodecyl derivative $\mathbf{8 d}$ was inactive, the bis-octyl derivative $\mathbf{8 e}$

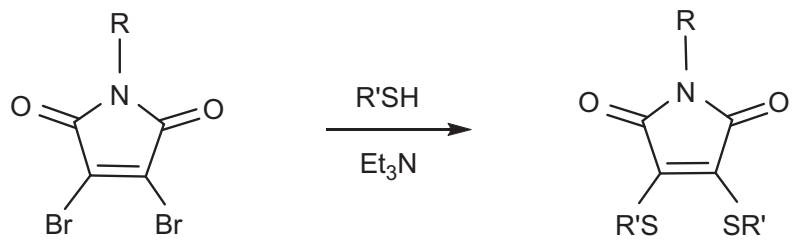

Scheme 1 Reaction of thiols with 3,4-dibromomaleimide. was a weak antibacterial and the bis-propylthio compound $8 \mathrm{f}$ displayed very high activity. It can be supposed that a correlation exists between lipophilicity of the maleimide substituents and antibacterial activity, and the high lipophilicity erodes the activity. To test this hypothesis, $\log \mathrm{P}$ (logarithm of partition coefficient between $n$ octanol and water) values were calculated for $\mathrm{N}$-methyl maleimide derivatives 11a-f and the calculated $\log \mathrm{P}$ values corroborate our postulation (Table 3 ).

In conclusion we have utilized, for the first time, bis-sulfide derivatives of $\mathrm{N}$-alkoxycarbonyl maleimide for versatile derivatisation of teicoplanin pseudoaglycon. It turned out that lipophilicity of substituents of the maleimide ring has strong influence on the antibacterial activity of these derivatives. Further synthetic tuning of these chemical structures hopefully will result in even more effective antibacterials.

\section{EXPERIMENTAL PROCEDURE}

\section{General information}

Maleimide and thiols $\mathbf{2} \mathbf{b}-\mathbf{2} \mathbf{g}$ were purchased from Sigma-Aldrich Chemical (St Louis, MO, USA). 2,3-Dibromomaleimide 1, 1,2:3,4-di-O-isopropylidene-6deoxy-6-thio- $\alpha$-D-galactopyranose $2 \mathrm{a}$ and teicoplanin pseudoaglycon 7 were prepared according to literature procedures. TLC analysis was performed on Kieselgel $60 \mathrm{~F}_{254}$ (Merck, Darmstadt, Germany) silica gel plates with visualization by immersing in ammonium-molibdate solution followed by heating or Pauly-reagent in the case of teicoplanin derivatives. Column chromatography was performed on silica gel 60 (Merck $0.063-0.200 \mathrm{~mm}$ ), flash column chromatography was performed on silica gel 60 (Merck $0.040-00.063 \mathrm{~mm}$ ). Organic solutions were dried over $\mathrm{MgSO}_{4}$ and concentrated under vacuum. The ${ }^{1} \mathrm{H}(400$ and $500 \mathrm{MHz})$ and ${ }^{13} \mathrm{C}$ NMR $(100.28,125.76 \mathrm{MHz})$ spectra were recorded with Bruker DRX-400 and Bruker Avance II 500 spectrometers. Chemical shifts are referenced to $\mathrm{Me}_{4} \mathrm{Si}$ or DSS (2,2-Dimethyl-2-silapentane-5sulfonate sodium salt) (0.00 p.p.m. for $\left.{ }^{1} \mathrm{H}\right)$ and to solvent signals $\left(\mathrm{CDCl}_{3}\right.$ :

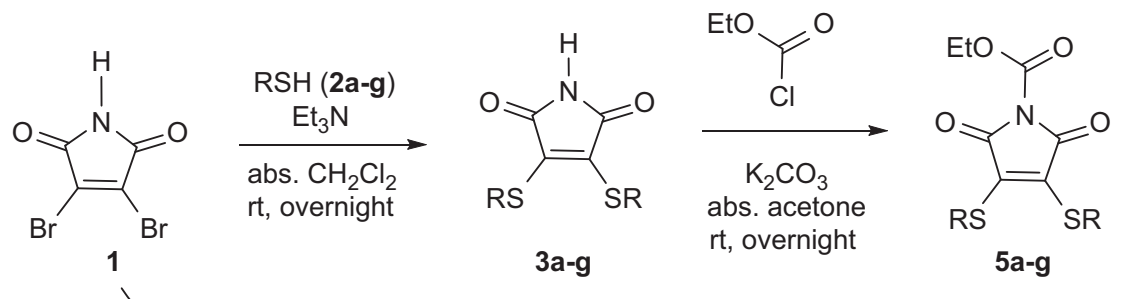

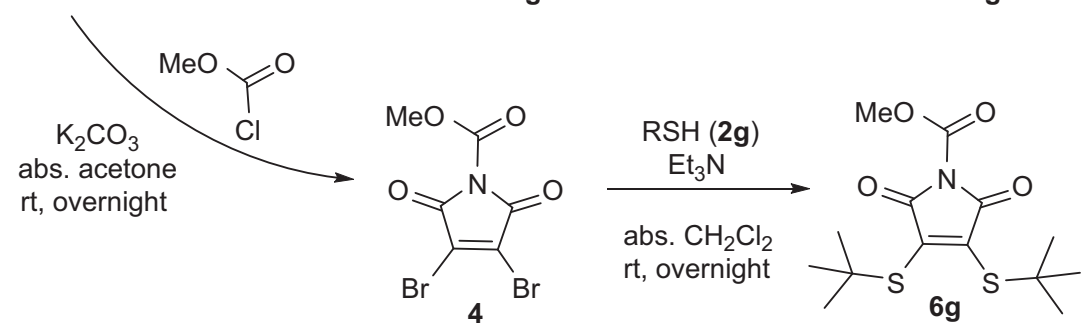

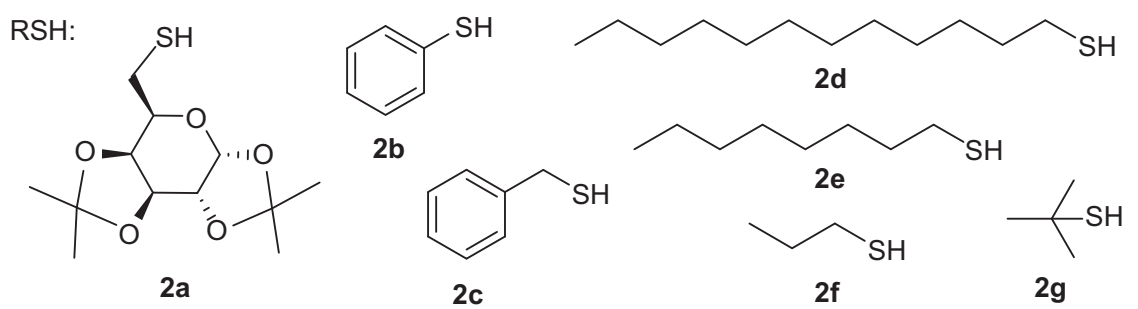

Scheme 2 Synthesis of $\mathrm{N}$-alkoxycarbonylated di-alkyl/arylthio-maleimide derivatives. 


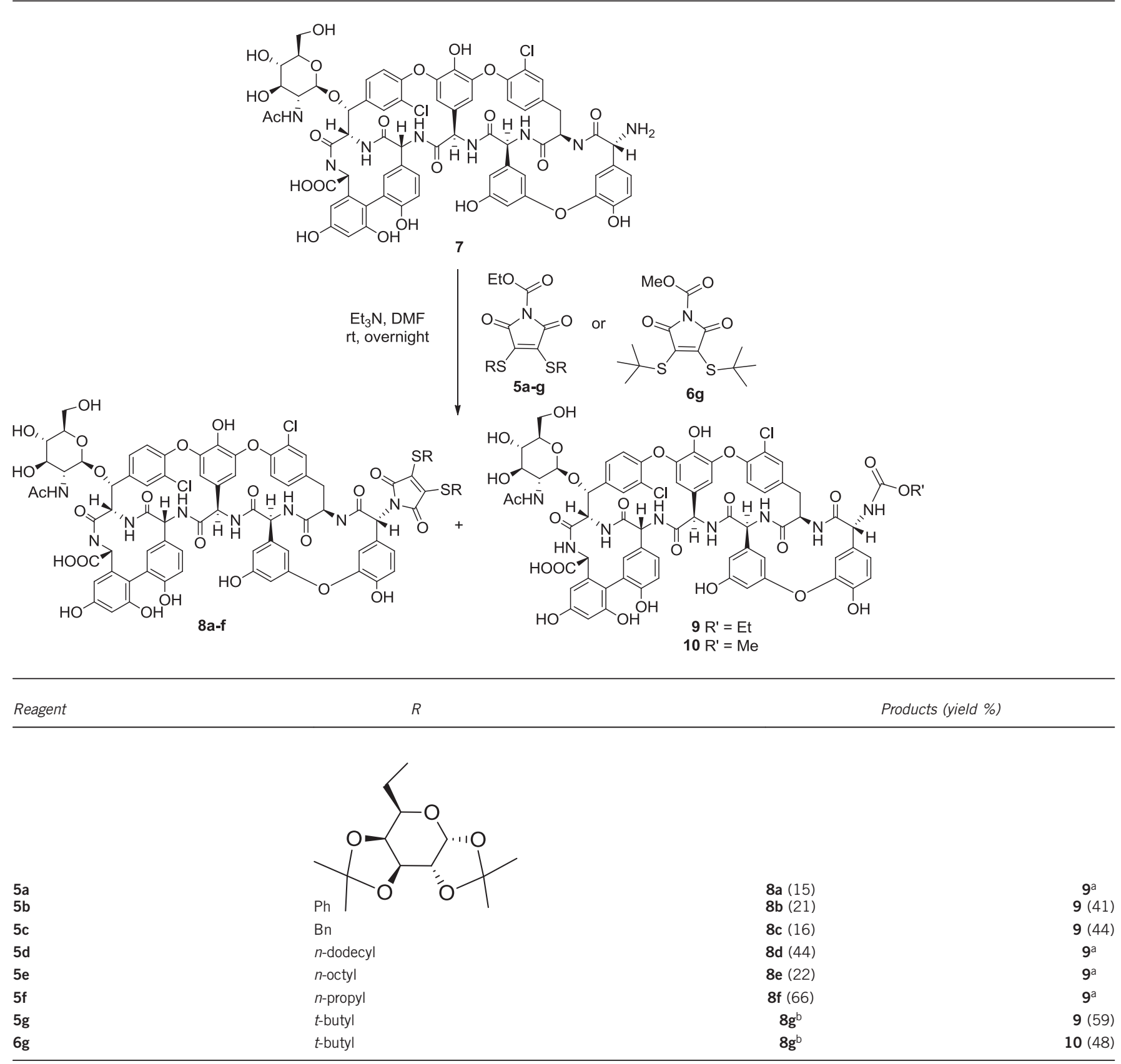

${ }^{\mathrm{a} F o r m a t i o n ~ w a s ~ o b s e r v e d ~(b a s e d ~ o n ~ T L C), ~ b u t ~ i t ~ w a s ~ n o t ~ i s o l a t e d . ~}$

bldentified by MS method but it could not be isolated in pure form.

77.00 p.p.m., DMSO-d 6 : 39.51 p.p.m. for ${ }^{13} \mathrm{C}$ ). MALDI-TOF MS analyses for the compounds $\mathbf{8 b}, \mathbf{8 c}, \mathbf{8 e}, \mathbf{9}$ and $\mathbf{1 0}$ were carried out in positive reflectron mode using a BIFLEX III mass spectrometer (Bruker, Bremen, Germany) equipped with delayed-ion extraction. In the case of $\mathbf{8 a}, \mathbf{8 d}$ and $\mathbf{8 f}$, MatrixAssisted Laser Desorption/Ionization Time-of-flight (MALDI-TOF) MS spectra were recorded by a Voyager-DE STR MALDI-TOF Biospectrometry Workstation (Applied Biosystems, Budapest, Hungary). 2,5-Dihydroxybenzoic acid was used as matrix and $\mathrm{CF}_{3} \mathrm{COONa}$ as cationising agent in DMF. Elemental analysis ( $\mathrm{C}, \mathrm{H}, \mathrm{S})$ was performed on an Elementar Vario MicroCube instrument. The antibacterial activity of $\mathbf{8 a}-\mathbf{f}, \mathbf{9}$ and $\mathbf{1 0}$ was tested against a panel of Gram-positive bacteria using broth microdilution method as described earlier. $^{24}$
General method A for preparation maleimide bis-sulfides (3a-3g) To a stirred solution of 2,3-dibromomaleimide ${ }^{23}$ (1.0 mmol) in $\mathrm{CH}_{2} \mathrm{Cl}_{2}(20 \mathrm{ml})$ $\mathrm{Et}_{3} \mathrm{~N}(2.0 \mathrm{mmol})$ and thiol $(2.1 \mathrm{mmol})$ were added under argon atmosphere and stirred for $3 \mathrm{~h}$ at room temperature. The reaction mixture was evaporated, and the crude product was purified by flash chromatography to give the desired compound.

General method B for preparation $N$-ethoxycarbonyl maleimide bis-sulfides (5a-5g)

To a stirred solution of maleimide bis-sulfide $(1.0 \mathrm{mmol})$ in dry acetone (20 ml) $\mathrm{K}_{2} \mathrm{CO}_{3}(1.2 \mathrm{mmol})$ and ethyl chloroformate $(1.2 \mathrm{mmol})$ were added 
Table 2 Antibacterial activity of compounds 7-10

\begin{tabular}{|c|c|c|c|c|c|c|c|c|c|c|}
\hline & Teicoplanin & 7 & $8 a$ & $8 b$ & $8 c$ & $8 d$ & $8 e$ & $8 f$ & 9 & 10 \\
\hline Staphylococcus aureus MSSA ATCC 29213 & $0.5 / 2$ & $2 / 32$ & $4 / 256$ & $2 / 16$ & $4 . / 32$ & $64 / 256$ & $8 / 64$ & $1 / 256$ & $16 / 128$ & $8 / 64$ \\
\hline Staphylococcus epidermidis biofilm ATCC 35984 & $2 / 32$ & $2 / 32$ & $1 / 256$ & $1 / 8$ & $0.5 / 2$ & $8 / 256$ & $1 / 8$ & $0.5 / 256$ & $4 / 32$ & $4 / 64$ \\
\hline Enterococcus faecalis ATCC 29212 & $2 / 64$ & $4 / 32$ & $4 / 256$ & $1 / 64$ & $0.5 / 64$ & $8 / 256$ & $8 / 256$ & $1 / 256$ & $8 / 256$ & $8 / 256$ \\
\hline Staphylococcus epidermidis mecA & $16 / 32$ & $1 / 32$ & $1 / 256$ & $2 / 16$ & $0.5 / 4$ & $8 / 256$ & $2 / 16$ & $0.5 / 256$ & $4 / 32$ & $8 / 64$ \\
\hline
\end{tabular}

Abbreviations: ATCC, American type culture collection; mecA, mecA gene expression in Staphylococcus; MRSA, methicillin resistant Staphylococcus aureus; MSSA, methicillin sensitive Staphylococcus aureus; van $A+$, vanA gene positive; $\operatorname{van} B+$, vanB gene positive.

Table 3 Calculated logP for $\mathrm{N}$-methyl maleimide derivatives $11 \mathrm{a}-\mathrm{f}$<smiles>[R5]C1=C([R5])C(=O)N(C)C1=O</smiles>

11a-f

\begin{tabular}{|c|c|c|}
\hline Compound & $R$ & $\log P$ \\
\hline $11 a$ & & 0.54 \\
\hline $11 b$ & $\mathrm{Ph}$ & 2.65 \\
\hline $11 \mathrm{c}$ & $\mathrm{Bn}$ & 2.78 \\
\hline $11 d$ & n-dodecyl & 8.48 \\
\hline $11 \mathrm{e}$ & n-octyl & 5.14 \\
\hline $11 \mathrm{f}$ & n-propyl & 0.97 \\
\hline
\end{tabular}

under argon atmosphere and stirred for $3 \mathrm{~h}$ at room temperature. The reaction mixture was diluted with $\mathrm{CH}_{2} \mathrm{Cl}_{2}$, filtered through a pad of Celite and evaporated. The crude product was used for further step without purification.

\section{General method $\mathrm{C}$ for the synthesis of teicoplanin pseudoaglycon derivatives $(\mathbf{8} \mathbf{a}-\mathbf{8 f})$}

To a stirred solution of teicoplanin pseudoaglycon ${ }^{16}(0.1 \mathrm{mmol})$ in dry DMF $(5 \mathrm{ml}) \mathrm{N}$-ethoxycarbonyl maleimide bis-sulfides $(0.14 \mathrm{mmol})$ and $\mathrm{Et}_{3} \mathrm{~N}(0.1$ $\mathrm{mmol}$ ) were added under argon atmosphere and stirred for overnight at room temperature. The reaction mixture was evaporated, and the crude product was purified by flash chromatography to give the desired compound.

Compound 3a. 2,3-Dibromomaleimide $(255 \mathrm{mg}, 1.0 \mathrm{mmol})$ was reacted with thiol $2 \mathrm{a}^{25}$ (580.4 mg, $2.1 \mathrm{mmol}$ ) according to general method A. The crude product was purified by silica gel chromatography in $n$-hexane:acetone $=8: 2$, to give $3 \mathrm{a}(550 \mathrm{mg}, 85 \%)$ as a yellow sirup. ${ }^{1} \mathrm{H} \mathrm{NMR}\left(400 \mathrm{MHz}, \mathrm{CDCl}_{3}\right) \delta 7.58$ $(1 \mathrm{H}, \mathrm{s}, \mathrm{NH}), 5.51\left(2 \mathrm{H}, \mathrm{d}, J_{1,2}=0.3 \mathrm{~Hz}, 2 \times \mathrm{H}-1\right), 4.62\left(2 \mathrm{H}, \mathrm{d}, J_{2,3}=8.0 \mathrm{~Hz}\right.$, $2 \times \mathrm{H}-2), 4.32-4.30(4 \mathrm{H}, \mathrm{m}, 2 \times \mathrm{H}-3,2 \times \mathrm{H}-4), 3.98-3.95(2 \mathrm{H}, \mathrm{m}, 2 \times \mathrm{H}-5)$,
3.57-3.36 (4H, m, $2 \times \mathrm{H}-6 \mathrm{a}, \mathrm{b}), 1.48,1.44,1.33,1.32\left(24 \mathrm{H}, 4 \times \mathrm{s}, 8 \times \mathrm{CH}_{3}\right.$-ip); ${ }^{13} \mathrm{C}$ NMR $\left(100 \mathrm{MHz}, \mathrm{CDCl}_{3}\right) \delta 165.8(2 \mathrm{C}, 2 \times \mathrm{C}=\mathrm{O}), 137.2,136.9(2 \mathrm{C}, \mathrm{C}=\mathrm{C})$, 109.5, 108.7 (4C, $4 \times \mathrm{C}_{\mathrm{q}}$-ip), 96.5 (2C, $\left.2 \times \mathrm{C}-1\right), 71.5,70.9,70.4,67.9$ (8C, skeleton carbons), $31.6(2 \mathrm{C}, 2 \times \mathrm{C}-6), 25.9,24.9,24.4\left(8 \mathrm{C}, 8 \times \mathrm{CH}_{3}\right)$; analysis calculated for $\mathrm{C}_{28} \mathrm{H}_{39} \mathrm{NO}_{12} \mathrm{~S}_{2}$ C 52.08, H 6.09, N 2.17, O 29.73, S 9.93. Found: C 51.99, H 6.08, S 9.90 .

Compound $3 \boldsymbol{b}$. 2,3-Dibromomaleimide $(255 \mathrm{mg}, 1.0 \mathrm{mmol})$ was reacted with thiophenol $\mathbf{2 b}(215 \mu \mathrm{l}, 2.1 \mathrm{mmol})$ according to general method A. The crude product was purified by silica gel chromatography in $n$-hexane: acetone $=8: 2$, to give $3 \mathbf{b}(310 \mathrm{mg}, 98 \%)$ as a yellow sirup. ${ }^{1} \mathrm{H}$ NMR $\left(400 \mathrm{MHz}, \mathrm{CDCl}_{3}\right) \delta 7.83(1 \mathrm{H}, \mathrm{s}, \mathrm{NH}), 7.29-7.17(10 \mathrm{H}, \mathrm{m}$, arom $) ;{ }^{13} \mathrm{C}$ NMR $\left(100 \mathrm{MHz}, \mathrm{CDCl}_{3}\right) \delta 166.5(2 \mathrm{C}, 2 \times \mathrm{C}=\mathrm{O}), 136.8(2 \mathrm{C}, \mathrm{C}=\mathrm{C}), 131.9$, 129.1, 128.6 (10C, arom), $128.9\left(2 \mathrm{C}, \mathrm{C}_{\mathrm{q}}\right.$ arom); analysis calculated for $\mathrm{C}_{16} \mathrm{H}_{11} \mathrm{NO}_{2} \mathrm{~S}_{2} \mathrm{C}$ 61.32, H 3.54, N 4.47, O 10.21, S 20.46. Found: C 61.15, H 3.53, S 20.39 .

Compound 3c. 2,3-Dibromomaleimide $(510 \mathrm{mg}, 2.0 \mathrm{mmol}$ ) was reacted with benzyl mercaptan $2 \mathrm{c}(490 \mu \mathrm{l}, 4.2 \mathrm{mmol})$ according to general method A. The crude product was purified by silica gel chromatography in $n$-hexane:acetone $=$ 8:2, to give $3 \mathrm{c}(460 \mathrm{mg}, 67 \%)$ as a yellow sirup. ${ }^{1} \mathrm{H} \mathrm{NMR}\left(400 \mathrm{MHz}, \mathrm{CDCl}_{3}\right) \delta$ $7.78(1 \mathrm{H}, \mathrm{s}, \mathrm{NH}), 7.29-7.26\left(10 \mathrm{H}, \mathrm{m}\right.$, arom), $4.42\left(4 \mathrm{H}, \mathrm{s}, 2 \times \mathrm{SCH}_{2}\right) ;{ }^{13} \mathrm{C} \mathrm{NMR}$ $\left(100 \mathrm{MHz}, \mathrm{CDCl}_{3}\right) \delta 175.3,166.3(2 \mathrm{C}, 2 \times \mathrm{C}=\mathrm{O}), 136.5(2 \mathrm{C}, \mathrm{C}=\mathrm{C}), 128.9$, $128.8,128.7,127.7$ (10C, arom), $36.2\left(2 \mathrm{C}, 2 \times \mathrm{SCH}_{2}\right)$; analysis calculated for $\mathrm{C}_{18} \mathrm{H}_{15} \mathrm{NO}_{2} \mathrm{~S}_{2} \mathrm{C} 63.32, \mathrm{H} 4.43, \mathrm{~N} 4.10$, O 9.37, S 18.78. Found: C 63.19, H 4.45, S 18.69 .

Compound 3d. 2,3-Dibromomaleimide $(510 \mathrm{mg}, 2.0 \mathrm{mmol}$ ) was reacted with dodecyl mercaptan $2 \mathrm{~d}(950 \mu \mathrm{l}, 4.2 \mathrm{mmol})$ according to general method A. The crude product was purified by silica gel chromatography in $n$-hexane:ethyl acetate $=9: 1$, to give $3 \mathbf{d}(670 \mathrm{mg}, 67 \%)$ as a yellow sirup. ${ }^{1} \mathrm{H} \mathrm{NMR}(400 \mathrm{MHz}$, $\left.\mathrm{CDCl}_{3}\right) \delta 7.55(1 \mathrm{H}, \mathrm{s}, \mathrm{NH}), 3.29-3.25\left(4 \mathrm{H}, \mathrm{m}, 2 \times \mathrm{SCH}_{2}\right), 1.64-1.25(40 \mathrm{H}, \mathrm{m}$, $\left.20 \times \mathrm{CH}_{2}\right), 0.89-0.86\left(6 \mathrm{H}, \mathrm{m}, 2 \times \mathrm{CH}_{3}\right) ;{ }^{13} \mathrm{C} \mathrm{NMR}\left(100 \mathrm{MHz}, \mathrm{CDCl}_{3}\right) \delta 165.8$ $(2 \mathrm{C}, 2 \times \mathrm{C}=\mathrm{O}), 136.4(2 \mathrm{C}, \mathrm{C}=\mathrm{C}), 31.5,31.4,30.2,29.3,29.1,28.9,28.7,28.1$ (20C, $20 \times \mathrm{CH} 2), 22.3\left(2 \mathrm{C}, 2 \times \mathrm{SCH}_{2}\right), 13.7\left(2 \mathrm{C}, 2 \times \mathrm{CH}_{3}\right)$. Analysis calculated for $\mathrm{C}_{28} \mathrm{H}_{51} \mathrm{NO}_{2} \mathrm{~S}_{2} \mathrm{C} 67.55, \mathrm{H} 10.33, \mathrm{~N} 2.81, \mathrm{O} 6.43, \mathrm{~S} 12.88$. Found: C $66.59, \mathrm{H}$ 10.23 , S 12.03 .

Compound 3 e. 2,3-Dibromomaleimide $(255 \mathrm{mg}, 1.0 \mathrm{mmol})$ was reacted with octyl mercaptan $2 \mathrm{e}(364 \mu \mathrm{l}, 2.1 \mathrm{mmol})$ according to general method A. The crude product was purified by silica gel chromatography in $n$-hexane:acetone $=$ 8:2, to give $3 \mathbf{e}(317 \mathrm{mg}, 82 \%)$ as a yellow sirup. ${ }^{1} \mathrm{H} \mathrm{NMR}\left(400 \mathrm{MHz}, \mathrm{CDCl}_{3}\right) \delta$ $7.71(1 \mathrm{H}, \mathrm{s}, \mathrm{NH}), 3.28\left(4 \mathrm{H}, \mathrm{t}, J=7.5 \mathrm{~Hz}, 2 \times \mathrm{SCH}_{2}\right), 1.69-1.60(8 \mathrm{H}, \mathrm{m}$, $\left.4 \times \mathrm{CH}_{2}\right), 1.43-1.27\left(20 \mathrm{H}, \mathrm{m}, 10 \times \mathrm{CH}_{2}\right), 0.88\left(6 \mathrm{H}, \mathrm{t}, J=6.8 \mathrm{~Hz}, 2 \times \mathrm{CH}_{3}\right) ;{ }^{13} \mathrm{C}$ NMR $\left(100 \mathrm{MHz}, \mathrm{CDCl}_{3}\right) \delta 166.3(2 \mathrm{C}, 2 \times \mathrm{C}=\mathrm{O}), 136.7(2 \mathrm{C}, \mathrm{C}=\mathrm{C}), 31.8$, $30.5,29.0,28.5\left(12 \mathrm{C}, 12 \times \mathrm{CH}_{2}\right), 22.6\left(2 \mathrm{C}, 2 \times \mathrm{SCH}_{2}\right), 14.0\left(2 \mathrm{C}, 2 \times \mathrm{CH}_{3}\right)$; analysis calculated for $\mathrm{C}_{20} \mathrm{H}_{35} \mathrm{NO}_{2} \mathrm{~S}_{2} \mathrm{C} 62.29, \mathrm{H} 9.15, \mathrm{~N} 3.63$, O 8.30, S 16.63. Found: C 61.03, H 9.08, S 16.08. 
Compound 3f. 2,3-Dibromomaleimide $(510 \mathrm{mg}, 2.0 \mathrm{mmol})$ was reacted with propyl mercaptane $2 \mathrm{f}(380 \mu \mathrm{l}, 4.2 \mathrm{mmol})$ according to general method A. The crude product was purified by silica gel chromatography in $n$-hexane:ethyl acetate $=9: 1$, to give $3 f(430 \mathrm{mg}, 87 \%)$ as a yellow sirup. ${ }^{1} \mathrm{H}$ NMR $\left(400 \mathrm{MHz}, \mathrm{CDCl}_{3}\right) \delta 7.77(1 \mathrm{H}, \mathrm{s}, \mathrm{NH}), 3.28-3.25(4 \mathrm{H}, \mathrm{m}$, $\left.2 \times \mathrm{SCH}_{2}\right), \quad 1.73-1.66\left(4 \mathrm{H}, \mathrm{m}, 2 \times \mathrm{CH}_{2}\right), \quad 1.06-1.02\left(6 \mathrm{H}, \mathrm{m}, 2 \times \mathrm{CH}_{3}\right)$; ${ }^{13} \mathrm{C}$ NMR $\left(100 \mathrm{MHz}, \mathrm{CDCl}_{3}\right) \delta 166.3(2 \mathrm{C}, 2 \times \mathrm{C}=\mathrm{O}), 137.2(2 \mathrm{C}, \mathrm{C}=\mathrm{C})$, $33.6\left(2 \mathrm{C}, 2 \times \mathrm{CH}_{2}\right), 23.8\left(2 \mathrm{C}, 2 \times \mathrm{SCH}_{2}\right), 13.1\left(2 \mathrm{C}, 2 \times \mathrm{CH}_{3}\right)$; analysis calculated for $\mathrm{C}_{10} \mathrm{H}_{15} \mathrm{NO}_{2} \mathrm{~S}_{2} \mathrm{C} 48.95, \mathrm{H} 6.16, \mathrm{~N}$ 5.71, O 13.04, S 26.14. Found: C 48.18, H 5.70, S 26.01 .

Compound 3g. 2,3-Dibromomaleimide (510 $\mathrm{mg}, 2.0 \mathrm{mmol}$ ) was reacted with t-butyl mercaptane $2 \mathrm{~g}$ ( $473 \mu \mathrm{l}, 4.2 \mathrm{mmol})$ according to general method A. The crude product was purified by silica gel chromatography in $n$-hexane:ethyl acetate $=9: 1$, to give $3 \mathbf{g}(432 \mathrm{mg}, 80 \%)$ as a yellow sirup. ${ }^{1} \mathrm{H} \mathrm{NMR}(400 \mathrm{MHz}$, $\left.\mathrm{CDCl}_{3}\right) \delta 8.09(1 \mathrm{H}, \mathrm{s}, \mathrm{NH}), 1.54\left(18 \mathrm{H}, \mathrm{s}, 6 \times \mathrm{CH}_{3}\right) ;{ }^{13} \mathrm{C} \mathrm{NMR}(100 \mathrm{MHz}$, $\left.\mathrm{CDCl}_{3}\right) \delta 166.9(2 \mathrm{C}, 2 \times \mathrm{C}=\mathrm{O}), 145.3(2 \mathrm{C}, \mathrm{C}=\mathrm{C}), 51.9\left(2 \mathrm{C}, 2 \times \mathrm{SC}_{\mathrm{q}}\right), 32.2$ (6C, $6 \times \mathrm{CH}_{3}$ ); analysis calculated for $\mathrm{C}_{12} \mathrm{H}_{19} \mathrm{NO}_{2} \mathrm{~S}_{2} \mathrm{C} 52.71, \mathrm{H}$ 7.00, N 5.12, O 11.70, S 23.46. Found: C 51.66, H 6.93, S 22.89.

Compound $6 \mathrm{~g}$. To a stirred solution of 2,3-dibromomaleimide $(0.255 \mathrm{~g}, 1.0$ $\mathrm{mmol})$ in tetrahydrofuran $(4 \mathrm{ml}) \mathrm{N}$-methylmorpholine $(76 \mu \mathrm{l}, 1.1 \mathrm{mmol})$ and methyl chloroformate $(85 \mu \mathrm{l}, 1.1 \mathrm{mmol})$ were added at $0^{\circ} \mathrm{C}$. When TLC $(n-$ hexane:acetone $=8: 2$ ) showed complete conversion of the starting material $\left(3 \mathrm{~h}\right.$ ), the reaction mixture was diluted with $\mathrm{CH}_{2} \mathrm{Cl}_{2}$, filtered through a pad of Celite and evaporated. The obtained crude $4(0.308 \mathrm{~g})$ was reacted, without purification, with $t$-butyl mercaptan $2 \mathrm{~g}(237 \mu \mathrm{l}, 2.1 \mathrm{mmol})$ according to general method A to give compound $6 \mathrm{~g}(0.150 \mathrm{~g})$. The crude product was used for further step without purification.

Compound $8 \boldsymbol{a}$. Teicoplanin pseudoaglycon $(140 \mathrm{mg}, 0.1 \mathrm{mmol})$ was reacted with compound $5 \mathbf{a}(100 \mathrm{mg}, 0.14 \mathrm{mmol})$ according to general method C. The crude product was purified by silica gel chromatography in toluene:methanol $=$
8:2, to give $\mathbf{8 a}(30 \mathrm{mg}, 15 \%)$ as a yellow powder. MALDI-TOF MS: [M+Na] ${ }^{+}=2051.39 \mathrm{~m} / \mathrm{z}$. Calcd for $\mathrm{C}_{94} \mathrm{H}_{94} \mathrm{Cl}_{2} \mathrm{~N}_{8} \mathrm{O}_{35} \mathrm{~S}_{2} \mathrm{Na} 2051.45 \mathrm{~m} / \mathrm{z}$.

Compound $8 \boldsymbol{b}$. Teicoplanin pseudoaglycon ( $140 \mathrm{mg} 0.1 \mathrm{mmol}$ ) was reacted with compound $5 \mathbf{b}(58 \mathrm{mg}, 0.14 \mathrm{mmol})$ according to general method $\mathrm{C}$. The crude product was purified by silica gel chromatography in toluene:methanol $=$ $8: 2$, to give $\mathbf{8 a}(35 \mathrm{mg}, 21 \%)$ as a yellow powder. MALDI-TOF MS: [M+Na] ${ }^{+}=1719.41 \mathrm{~m} / z$. Calcd for $\mathrm{C}_{82} \mathrm{H}_{66} \mathrm{Cl}_{2} \mathrm{~N}_{8} \mathrm{O}_{25} \mathrm{~S}_{2} \mathrm{Na} 1719.29 \mathrm{~m} / \mathrm{z}$.

Compound $8 \boldsymbol{c}$. Teicoplanin pseudoaglycon $(140 \mathrm{mg}, 0.1 \mathrm{mmol})$ was reacted with compound $5 \mathrm{c}(41 \mathrm{mg}, 0.14 \mathrm{mmol})$ according to general method C. The crude product was purified by silica gel chromatography in toluene:methanol $=$ $7: 3$, to give $8 \mathrm{c}(27 \mathrm{mg}, 16 \%)$ as a yellow powder. MALDI-TOF MS: [M+Na] ${ }^{+}=1747.47 \mathrm{~m} / \mathrm{z}$. Calcd for $\mathrm{C}_{84} \mathrm{H}_{70} \mathrm{Cl}_{2} \mathrm{~N}_{8} \mathrm{O}_{25} \mathrm{~S}_{2} \mathrm{Na} 1747.32 \mathrm{~m} / \mathrm{z}$

Compound $8 \boldsymbol{d}$. Teicoplanin pseudoaglycon $(140 \mathrm{mg}, 0.1 \mathrm{mmol})$ was reacted with compound $5 \mathbf{d}(74 \mathrm{mg}, 0.14 \mathrm{mmol})$ according to general method C. The crude product was purified by silica gel chromatography in toluene:methanol $=$ 9:1, to give $8 \mathbf{d}(85 \mathrm{mg}, 44 \%)$ as a yellow powder. MALDI-TOF MS: [M+Na] ${ }^{+}=1903.66 \mathrm{~m} / \mathrm{z}$. Calcd for $\mathrm{C}_{94} \mathrm{H}_{106} \mathrm{Cl}_{2} \mathrm{~N}_{8} \mathrm{O}_{25} \mathrm{~S}_{2} \mathrm{Na} 1903.60 \mathrm{~m} / \mathrm{z}$.

Compound $8 \boldsymbol{e}$. Teicoplanin pseudoaglycon $(140 \mathrm{mg}, 0.1 \mathrm{mmol})$ was reacted with compound $5 \mathbf{e}(69 \mathrm{mg}, 0.14 \mathrm{mmol})$ according to general method C. The crude product was purified by silica gel chromatography in toluene:methanol $=$ 8:2, to give $8 \mathbf{d}(38 \mathrm{mg}, 22 \%)$ as a yellow powder. MALDI-TOF MS: [M+Na] ${ }^{+}=1791.64 \mathrm{~m} / \mathrm{z}$. Calcd for $\mathrm{C}_{86} \mathrm{H}_{90} \mathrm{Cl}_{2} \mathrm{~N}_{8} \mathrm{O}_{25} \mathrm{~S}_{2} \mathrm{Na} 1791.47 \mathrm{~m} / \mathrm{z}$.

Compound $8 f$. Teicoplanin pseudoaglycon $(140 \mathrm{mg}, 0.1 \mathrm{mmol})$ was reacted with compound $\mathbf{5 f}(40 \mathrm{mg}, 0.14 \mathrm{mmol})$ according to general method $\mathrm{C}$. The crude product was purified by silica gel chromatography in toluene:methanol = 9:1, to give $\mathbf{8 d}(110 \mathrm{mg}, 66 \%)$ as a yellow powder. MALDI-TOF MS: [M+Na] ${ }^{+}=1651.02 \mathrm{~m} / z$. Calcd for $\mathrm{C}_{76} \mathrm{H}_{70} \mathrm{Cl}_{2} \mathrm{~N}_{8} \mathrm{O}_{25} \mathrm{~S}_{2} \mathrm{Na} 1651.32 \mathrm{~m} / \mathrm{z}$.

Compound 9. Teicoplanin pseudoaglycon $(140 \mathrm{mg}, 0.1 \mathrm{mmol})$ was reacted with compound $5 \mathrm{~g}(49 \mathrm{mg}, 0.14 \mathrm{mmol})$ according to general method C. The

Table $4{ }^{1} \mathrm{H}$ and ${ }^{13} \mathrm{C}$ NMR data for compounds $8 \mathrm{a}, 8 \mathrm{~b}, 8 \mathrm{c}$ and $8 \mathrm{~d}$ (chemical shifts in ppm)

\begin{tabular}{|c|c|c|c|c|c|c|c|c|}
\hline Assignment & $8 a^{13} C$ & $8 a^{1} H$ & $8 b^{13} C$ & $8 b^{1} H$ & $8 c^{13} C$ & $8 c^{1} H$ & $8 d^{13} C$ & $8 d^{1} H$ \\
\hline$x 1$ & 64.8 & 7.05 & 64.8 & 7.07 & 64.9 & 7.06 & 64.6 & 7.05 \\
\hline$x 2$ & 55.6 & 4.98 & 55.9 & 4.98 & 55.9 & 4.99 & 55.5 & 4.98 \\
\hline$x 3$ & 59.2 & 5.32 & 59.1 & 5.29 & 59.2 & 5.33 & 59.1 & 5.36 \\
\hline$x 4$ & 54.8 & 5.59 & 54.8 & 5.58 & 54.9 & 5.59 & 54.8 & 5.64 \\
\hline$z 6$ & 76.8 & 5.40 & 76.2 & 5.45 & 76.7 & 5.42 & 76.3 & 5.42 \\
\hline $2 f$ & 131.5 & 7.68 & 131.6 & 7.67 & 131.5 & 7.65 & 131.8 & 7.69 \\
\hline $3 b$ & 109.7 & 6.32 & 109.7 & 6.28 & 110.2 & 6.32 & 110.0 & 6.39 \\
\hline $4 b$ & 107.9 & 5.57 & 108.2 & 5.53 & 108.3 & 5.55 & 108.2 & 5.55 \\
\hline $4 f$ & 104.6 & 5.07 & 104.8 & 5.06 & 104.9 & 5.07 & 104.9 & 5.06 \\
\hline $5 b$ & 136.3 & 7.09 & 136.6 & 7.09 & 136.6 & 7.09 & 136.5 & 7.11 \\
\hline GlcNAc 1 & 98.4 & 4.40 & 98.8 & 4.39 & 99.0 & 4.36 & 99.0 & 4.39 \\
\hline Maleimide 2 & 165.3 & & 165.4 & & 165.4 & & 165.5 & \\
\hline Maleimide 3 & 135.3 & & 135.3 & & 136.8 & & 134.2 & \\
\hline Maleimide 4 & 135.3 & & 135.3 & & 136.8 & & 134.2 & \\
\hline Maleimide 5 & 165.3 & & 165.4 & & 165.4 & & 165.5 & \\
\hline $\mathrm{SCH}_{2}$ & & & & & 35.5 & $4.42-4.37$ & 31.5 & $3.33-3.21$ \\
\hline$\alpha$-Galp 1 & 95.7 & 5.41 & & & & & & \\
\hline$\alpha$-Galp 2 & 69.7 & 4.30 & & & & & & \\
\hline$\alpha$-Galp 3 & 70.2 & 4.60 & & & & & & \\
\hline$\alpha$-Galp 4 & 70.9 & 4.22 & & & & & & \\
\hline$\alpha$-Galp 5 & 67.2 & 3.82 & & & & & & \\
\hline$\alpha$-Galp 6 & 31.2 & $3.37-3.27$ & & & & & & \\
\hline $\mathrm{P}^{\mathrm{P}}-\mathrm{C}_{\mathrm{q}}$ & $108.8 ; 108.6$ & & & & & & & \\
\hline${ }_{i}-\mathrm{CH}_{3}$ & 31.5-24.2 & $1.40-1.23$ & & & & & & \\
\hline $\mathrm{Ph}$ & & & $131.0-128.18$ & $7.29-7.15$ & & & & \\
\hline
\end{tabular}


Table $5{ }^{1} \mathrm{H}$ and ${ }^{13} \mathrm{C}$ NMR data for compounds $8 \mathrm{e}, 8 \mathrm{f}, 9$ and 10 (chemical shifts in p.p.m.)

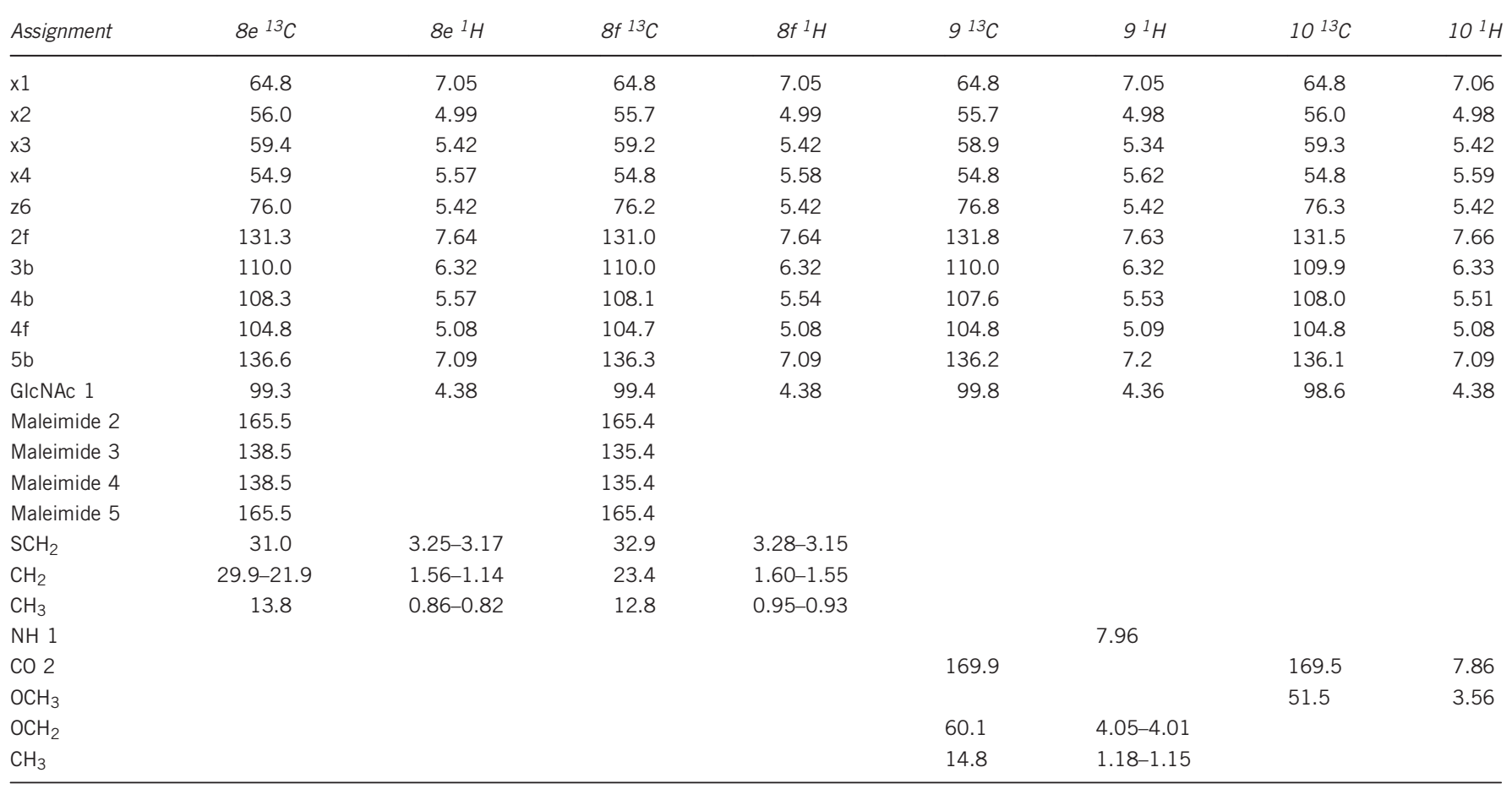

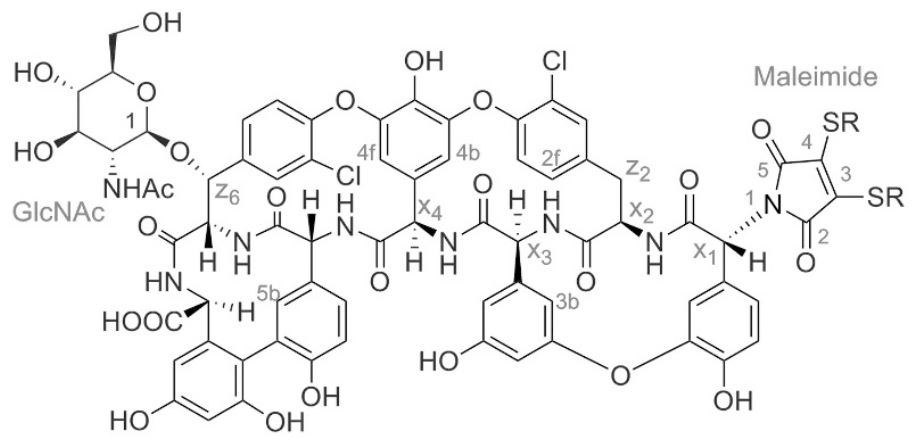

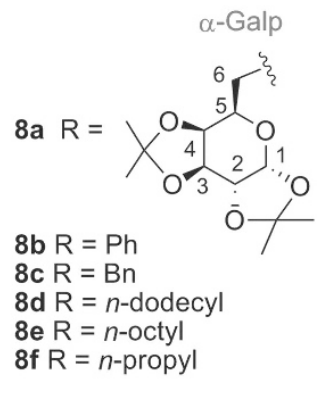

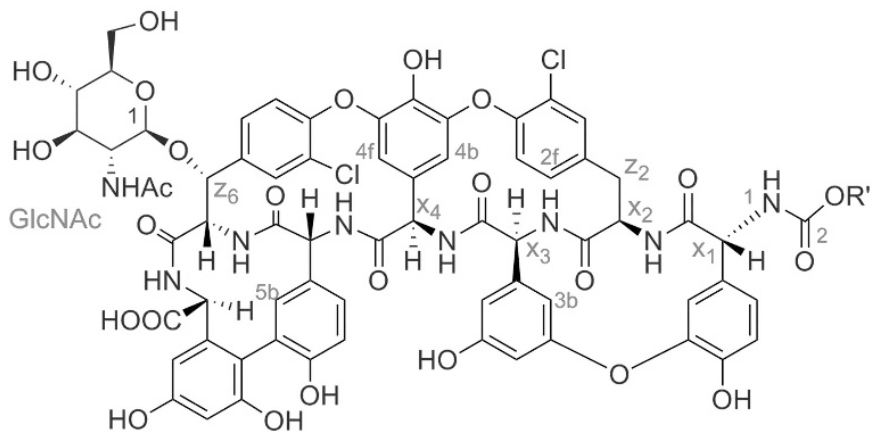

$9 \mathrm{R}^{\prime}=\mathrm{Et}$

$10 \mathrm{R}^{\prime}=\mathrm{Me}$

Figure 1 Structure and numbering for compounds $8 \mathbf{a}-\mathbf{f}, \mathbf{9}$ and 10. A full color version of this figure is available at The Journal of Antibiotics journal online.

crude product was purified by silica gel chromatography in toluene:methanol $=$ 9:1, to give $9(87 \mathrm{mg}, 59 \%)$ as a yellow powder. MALDI-TOF MS: $[\mathrm{M}+\mathrm{Na}]^{+}=$ $1495.34 \mathrm{~m} / z$. Calcd for $\mathrm{C}_{69} \mathrm{H}_{62} \mathrm{Cl}_{2} \mathrm{~N}_{8} \mathrm{O}_{25} \mathrm{Na} 1495.31 \mathrm{~m} / \mathrm{z}$.
Compound 10. Teicoplanin pseudoaglycon $(210 \mathrm{mg}, \quad 0.15 \mathrm{mmol})$ was reacted with compound $6 \mathrm{~g}(70 \mathrm{mg}, 0.21 \mathrm{mmol})$ according to general method C. The crude product was purified by silica gel chromatography 
in toluene:methanol $=8: 2$, to give $10(120 \mathrm{mg}, 48 \%)$ as a yellow powder. MALDI-TOF MS: $[\mathrm{M}+\mathrm{Na}]^{+}=1481.51 \mathrm{~m} / z$. Calcd for $\mathrm{C}_{68} \mathrm{H}_{60} \mathrm{Cl}_{2} \mathrm{~N}_{8} \mathrm{O}_{25} \mathrm{Na}$ $1481.29 \mathrm{~m} / \mathrm{z}$

\section{NMR analysis}

The ${ }^{1} \mathrm{H}$ and ${ }^{13} \mathrm{C}$ NMR data of the teicoplanin derivatives $\mathbf{8} \mathbf{a}-\mathbf{f}, \mathbf{9}$ and $\mathbf{1 0}$ are collected in Tables 4 and 5 . The spectra were recorded at $500.13 / 125.76 \mathrm{MHz}$ frequencies, respectively, at $300 \mathrm{~K}$, using DMSO- $\mathrm{d}_{6}$, as solvent. Numbering atoms in teicoplanin derivatives are given in Figure 1. Signal assignments were aided by $2 \mathrm{D}$ HSQC, TOCSY (15 and $60 \mathrm{~ms}$ mixing times) and HMBC (60 ms mixing time) experiments.

\section{ACKNOWLEDGEMENTS}

This research was supported by the European Union and the State of Hungary, co-financed by the European Social Fund in the framework of TÁMOP 4.2.4.A/ 2-11-1-2012-0001 'National Excellence Program'. The study was also supported by the Hungarian Research Fund (OTKA K 109208 and ANN 110821) and by the University of Debrecen (bridging fund to $\mathrm{PH}$ ).

1 Kahne, D., Leimkuhler, C., Lu, W. \& Walsh, C. Glycopeptide and lipoglycopeptide antibiotics. Chem. Rev. 105, 425-448 (2005).

2 von Nussbaum, F., Brands, M., Hinzen, B., Weigand, S. \& Habich, D. Antibacterial natural products in medicinal chemistry-exodus or revival? Angew. Chem. Int. Ed. Engl. 45, 5072-5129 (2006).

3 Kristóf, K. et al. Significance of methicillin-teicoplanin resistant Staphylococcus haemolyticus in bloodstream infections in patients of the Semmelweis University hospitals in Hungary. Eur. J. Clin. Microbiol. Infect. Dis. 30, 691-699 (2011).

4 Xu, H.-W., Shang-Shang Qin, S.-S. \& Liu, H.-M. New synthetic antibiotics for the treatment of enterococcus and campylobacter infection. Current Topics Med. Chem. 14, 21-39 (2014).

5 James, R. C., Pierce, J. G., Okano, A., Xie, J. \& Boger, D. L. Redesign of glycopeptide antibiotics: Back to the future. ACS Chem. Biol. 7, 797-804 (2012).

6 Ashford, P.-A. \& Bew, S. P. Recent advances in the synthesis of new glycopeptide antibiotics. Chem. Soc. Rev. 41, 957-978 (2012).

7 Cooper, R. D. et al. Reductive alkylation of glycopeptide antibiotics: synthesis and antibacterial activity. J. Antibiot. 49, 575-581 (1996).
8 Judice, J. K. \& Pace, J. L. Semi-synthetic glycopeptide antibacterials. Bioorg. Med. Chem. Lett. 13, 4165-4168 (2003).

9 Malabarba, A. \& Ciabatti, R. Glycopeptide derivatives. Curr. Med. Chem. 8, 1759-1773 (2001).

10 Sztaricskai, F. et al. N-glycosylthioureido aglyco-ristocetins without platelet aggregation activity. J. Antibiot. 60, 529-533 (2007).

11 Pinter, G. et al. Click reaction synthesis of carbohydrate derivatives from ristocetin aglycon with antibacterial and antiviral activity. Bioorg. Med. Chem. Lett. 20, 2713-2717 (2010).

12 Sipos, A. et al. Synthesis of isoindole and benzoisoindole derivatives of teicoplanin pseudoaglycon with remarkable antibacterial and antiviral activities. Bioorg. Med. Chem. Lett. 22, 7092-7096 (2012).

13 Sipos, A. et al. Synthesis of fluorescent ristocetin aglycon derivatives with remarkable antibacterial and antiviral activities. Eur. J. Med. Chem. 56, 361-367 (2012).

14 Naesens, L. et al. Anti-influenza virus activity and structure-activity relationship of aglycoristocetin derivatives with cyclobutenedione carrying hydrophobic chains. Antiviral Res. 82, 89-94 (2009).

15 Vanderlinden, E. et al. Intracytoplasmic trapping of influenza virus by a lipophilic derivative of aglycoristocetin. J. Virol. 86, 9416-9431 (2012).

16 Pintér, G. et al. A diazo transfer-click reaction route to new, lipophilic teicoplanin and ristocetin aglycon derivatives with high antibacterial and anti-influenza virus activity: an aggregation and receptor binding study. J. Med. Chem. 52, 6053-6061 (2009).

17 Bereczki, I. et al. Semisynthetic teicoplanin derivatives as new influenza virus binding inhibitors: synthesis and antiviral studies. Bioorg. Med. Chem Lett. 24, 3251-3254 (2014).

18 Smith, M. E. B. et al. Protein modification, bioconjugation, and disulfide bridging using bromomaleimides. J. Am. Chem. Soc. 132, 1960-1965 (2010).

19 Schumacher, F. F. et al. In situ maleimide bridging of disulfides and a new approach to protein PEGylation. Bioconj. Chem. 22, 132-136 (2011).

20 Ryan, C. P. et al. Tunable reagents for multi-functional bioconjugation: reversible or permanent chemical modification of proteins and peptides by control of maleimide hydrolysis. Chem. Commun. 47, 5452-5454 (2011).

21 Schumacher, F. F. et al. Next generation maleimides enable the controlled assembly of antibody-drug conjugates via native disulfide bond bridging. Org. Biomol. Chem. 12, 7261-7269 (2014).

22 Castañeda, L. et al. A mild synthesis of $\mathrm{N}$-functionalised bromomaleimides, thiomaleimides and bromopyridazinediones. Tetrahedron Lett. 54, 3493-3495 (2003).

23 Marminon, C. et al. Syntheses and antiproliferative activities of rebeccamycin analogues bearing two 7-azaindole moieties. Bioorg. Med. Chem. 11, 679-687 (2003).

24 Sztaricskai, F. et al. A new series of glycopeptide antibiotics incorporating a squaric acid moiety. J. Antibiot. 59, 564-582 (2006).

25 Martins Alho, M. A., D'Accorso, N. B. \& Thiel, I. M. E. Syntheses of some 6-Sheterocyclic derivatives of 1,2:3,4-di-O-isopropylidene- $\alpha$-D-galactopyranose. J. Heterocyclic Chem. 33, 1339-1343 (1996). 